\title{
Jerarquización de la evidencia. Niveles de evidencia y grados de recomendación de uso actual
}

\author{
Carlos Manterola, Claudia Asenjo-Lobos y Tamara Otzen
}

\section{Hierarchy of evidence. Levels of evidence and grades of recommendation from current use}

There are multiple proposals and classifications that hierarchize evidence, which may confuse those who are dedicated to generate it both in health technology assessments, as for the development of clinical guidelines, etc. The aim of this manuscript is to describe the most commonly used classifications of levels of evidence and grades of recommendation, analyzing their main differences and applications so that the user can choose the one that better suits your needs and take this health decisions basing their practice on the best available evidence. A systematic literature search was performed in PubMed and MEDLINE databases and in Google, Yahoo and Ixquick search engines. A wealth of information concerning levels of evidence and degrees recommendation was obtained. It was summarized the information of the 11 proposals more currently used (CTFPHC, Sackett, USPSTF, CEBM, GRADE, SIGN, NICE, NHMRC, PCCRP, ADA y ACCF/AHA), between which it emphasizes the GRADE WORKING GROUP, incorporated by around 90 national and international organizations such as the World Health Organization, The Cochrane Library, American College of Physicians, American Thoracic Society, UpToDate, etc.; and locally by the Ministry of Health to create clinical practice guidelines.

Key words: "Evidence-Based Practice"[Mesh], "Evidence-Based Medicine", levels of evidence, grades of recommendation, clinical recommendation.

Palabras clave: Práctica clínica basada en la evidencia; medicina basada en la evidencia niveles de evidencia, grados de recomendación, recomendación clínica.

\section{Introducción}

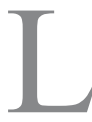

os primeros indicios de la práctica de la medicina basada en la evidencia (MBE), se remontan a los tiempos del emperador Qianlong, cuando se utilizaba el método "kaozheng" que representa la "práctica de la investigación probatoria" $"$. Desde entonces, numerosas instancias aisladas se fueron desarrollando hasta que en la década de los ochenta, el grupo de la Universidad McMaster, liderado por David Sackett, desarrolló los principios de la enseñanza y práctica de la MBE que rigen hasta hoy, y que se pueden resumir como "integrar la experiencia clínica individual con la mejor evidencia disponible a partir de la investigación sistemática"3-5.

En la búsqueda de la mejor evidencia disponible, es necesario otorgar un valor jerárquico a la evidencia disponible, con el objetivo de tener una guía para decidir si aplicar o no una determinada intervención, tratamiento o procedimiento; a partir de la cual se pueda emitir una recomendación basada en la solidez de la evidencia que la respalda.

La primera jerarquización de la evidencia fue formulada por la Canadian Task Force on the Periodic Health Examination en 1979. Ésta, se desarrolló para la evaluación de medidas preventivas. Fue adaptada posteriormente por la U.S. Preventive Services Task Force (USPSTF) en 1984, quienes organizaron los niveles de evidencia (NE) y grados de recomendación (GR) para sujetos asintomáticos, indicando cuáles procedimientos eran los más adecuados y cuáles debían ser evitados.

En general, las clasificaciones se basan en los diseños de los estudios de donde proviene la evidencia, asumiendo que algunos de ellos están sujetos a más sesgos que otros; y por ende, justifican más débilmente las decisiones clínicas. Por otro lado, el análisis constante de la evidencia disponible desde la perspectiva de los diferentes escenarios clínicos, permite establecer GR para el ejercicio de procedimientos diagnósticos, terapéuticos, preventivos, económicos en salud, etc.; e indican la forma de valorar la evidencia disponible en función de etiología, daño, morbilidad y complicaciones; pronóstico, historia natural y curso clínico, etc. de una enfermedad o evento de interés ${ }^{6}$.

Ha existido una proliferación de propuestas y clasificaciones que jerarquizan la evidencia; y junto con ello, sus respectivos GR. Ambos, pueden confundir a quienes se dedican a generar la evidencia a través del reporte de resultados por medio de artículos primarios y revisiones sistemáticas; así como de la realización de documentos de resumen de la evidencia (overviews), elaboración de guías clínicas, evaluaciones de tecnología sanitaria, etc.
Universidad de La Frontera,

Temuco, Chile.

Departamento de Cirugía y Traumatología (CM). Universidad de Concepción Concepción, Chile.

Centro de Rehabilitación Avanzada e Implantología-C RAl- (CA). Universidad Autónoma de Chile. Escuela de Psicología (TO). Conflictos de interés: Ninguno. Financiamiento: Programa de Doctorado en Ciencias Médicas, Universidad de La Frontera.

Recibido: 30 de marzo de 2014 Aceptado: 31 de mayo de 2014

Correspondencia a: Carlos Manterola Delgado carlos.manterola@ufrontera.cl 
Esto es debido a las diferencias y a la complejidad de las escalas de valoración existentes. Adicionalmente, el lenguaje utilizado para expresar la información no es de uso rutinario en la práctica cotidiana ${ }^{6}$, lo que determina aún más confusión e incertidumbre respecto de cuál aplicar.

El objetivo de este artículo es actualizar la información y describir las clasificaciones más utilizadas para valorar la evidencia en el ámbito de la salud, analizando sus principales diferencias y aplicaciones para que el usuario pueda elegir la que mejor se adapte a sus necesidades y tomar de este modo decisiones sanitarias basando su práctica en la mejor evidencia disponible.

\section{Clasificación de la evidencia y tipos de estudio}

En las dos últimas décadas se ha producido un incremento significativo de la investigación clínica basada en la evidencia, como pilar fundamental en la toma de decisiones para los cuidados en salud. Sin embargo, no todos los conocimientos provenientes de artículos científicos tienen el mismo impacto o valor sobre la toma de decisiones; por tal razón, se debe aplicar un método riguroso para compilar la evidencia científica en torno a una pregunta; analizar de forma crítica los artículos científicos de los que disponemos para responder a la interrogante en cuestión, valorando la validez interna (metodología empleada y riesgo de sesgos), el impacto

\begin{tabular}{|c|l|}
\hline $\begin{array}{c}\text { Grados de } \\
\text { recomendación }\end{array}$ & Interpretación \\
\hline A & Existe buena evidencia para recomendar la intervención clínica de prevención \\
\hline B & Existe evidencia moderada para recomendar la intervención clínica de prevención \\
\hline C & $\begin{array}{l}\text { La evidencia disponible es contradictoria y no permite hacer recomendaciones a } \\
\text { favor o en contra de la intervención clínica preventiva; sin embargo, otros factores } \\
\text { podrían influenciar en la decisión }\end{array}$ \\
\hline D & $\begin{array}{l}\text { Existe evidencia moderada para NO recomendar la intervención clínica de pre- } \\
\text { vención }\end{array}$ \\
\hline E & Existe buena evidencia para NO recomendar la intervención clínica de prevención \\
\hline I & $\begin{array}{l}\text { Existe evidencia insuficiente (cualitativa y cuantitativamente) para hacer una } \\
\text { recomendación; sin embargo, otros factores podrían influenciar en la decisión }\end{array}$ \\
\hline
\end{tabular}

Figura 1. Grados de recomendación para las intervenciones de prevención (CTFPHC).

\begin{tabular}{|c|l|}
\hline $\begin{array}{c}\text { Niveles de } \\
\text { evidencia }\end{array}$ & Interpretación \\
\hline I & Evidencia existente surge a partir de EC CON asignación aleatoria. \\
\hline II-1 & Evidencia existente surge a partir de EC SIN asignación aleatoria. \\
\hline II-2 & $\begin{array}{l}\text { Evidencia existente surge a partir de estudios de cohortes, y de casos y controles, } \\
\text { idealmente realizados por más de un centro o grupo de investigación. }\end{array}$ \\
\hline II-3 & $\begin{array}{l}\text { Evidencia existente surge a partir de comparaciones en el tiempo o entre distintos } \\
\text { centros, con o sin la intervención; podrían incluirse resultados provenientes de estu- } \\
\text { dios SIN asignación aleatoria. }\end{array}$ \\
\hline III & $\begin{array}{l}\text { Evidencia existente surge a partir de la opinión de expertos, basados en la experiencia } \\
\text { clínica; estudios descriptivos o informes de comités de expertos. }\end{array}$ \\
\hline
\end{tabular}

Figura 2. Niveles de evidencia e interpretación de los tipos de estudio para intervenciones de prevención (CTFPHC). de los resultados y la validez externa del artículo (posible reproducibilidad de los resultados en la población que nos interesa). Previo a ello, el o los artículos seleccionados deben ser asignados a algún escenario clínico (tratamiento, prevención, etiología, daño, pronóstico e historia natural, diagnóstico diferencial, prevalencia, tamizaje, estudios económicos y análisis de decisión), a propósito de lo cual se elegirá la guía de usuario o pauta de lectura crítica correspondiente con la que se evaluará cada artículo.

Una vez identificado el escenario en el que corresponde catalogar al artículo (en ocasiones, puede ser asignado a más de uno), se aplica según el tipo de diseño del estudio en cuestión, la propuesta de NE y GR. Por lo anteriormente expuesto, es fundamental identificar el escenario y los diseños de investigación para poder valorar la evidencia utilizando alguna de las clasificaciones existentes.

Realizamos una búsqueda sistemática de la literatura en las bases de datos PubMed y MEDLINE y en los buscadores Google, Yahoo e Ixquick, utilizando los términos: medicina basada en la evidencia, evidencia, niveles de evidencia, grados de recomendación, fuerza de recomendación; en idiomas inglés y castellano. Obtuvimos una gran cantidad de información referente a niveles de evidencia y grados de recomendación, para finalmente resumir la información de 11 de las propuestas más utilizadas en la actualidad (CTFPHC, Sackett, USPSTF, CEBM, GRADE, SIGN, NICE, NHMRC, PCCRP, ADA y ACCF/ AHA), que son las que se describen a continuación.

\section{Canadian Task Force on Preventive Health Care}

La Canadian Task Force on Preventive Health Care (CTFPHC), fue elaborada por la Public Health Agency of Canada (PHAC) para desarrollar guías de práctica clínica que respaldasen las acciones de salud preventiva ${ }^{7}$.

En sus inicios, este grupo hizo énfasis en el tipo de diseño utilizado y la calidad de los estudios publicados, basándose en los siguientes elementos: Un orden para los GR establecido por letras del abecedario donde las letra A y B indican que existe evidencia para ejercer una acción (se recomienda hacer); D y E indican que no debe llevarse a cabo la maniobra o acción determinada (se recomienda no hacer); la letra $\mathrm{C}$, indica que la evidencia es "contradictoria"; y la letra I, indica insuficiencia en calidad y cantidad de evidencia disponible para establecer una recomendación (Figura 1) ${ }^{8}$. Y, NE clasificados según el diseño de los estudios de I a III, disminuyendo en calidad según se acrecienta numéricamente, para lo cual son clasificados según validez interna o calidad metodológica del estudio (Figuras 2 y 3 ).

Adicionalmente, CTFPHC se apoya en el sistema GRADE (Grading of Recommendations Assessment, Development and Evaluation $)^{9,10}$, para evaluar la calidad de la evidencia y realizar recomendaciones en el ámbito de la prevención. 


\section{Clasificación de Sackett}

Esta sistematización propuesta por el epidemiólogo David L. Sackett, jerarquiza la evidencia en niveles que van de 1 a 5 ; siendo el nivel 1 la "mejor evidencia" y el nivel 5 la "peor, la más mala o la menos buena" (Figura 4) ${ }^{11}$.

Las recomendaciones en apoyo de una intervención pueden ser generadas en base a estos cinco NE. De este modo, estudios nivel 1 conllevan a un GR A: resultados apoyados por estudios; nivel 2, reciben un GR B y las recomendaciones $\mathrm{C}$ se asignan a los resultados apoyados por estudios nivel 3, 4 ó 5. Así, el nivel indica el grado de certeza, generado por la fuerza de la evidencia. Grado A: Las conclusiones se generan a partir de la evidencia más fuerte de la investigación y por tanto son los más definitivos. Grado B: Las conclusiones se basan en pruebas más débiles y sólo son orientativas. Grado C: Las conclusiones se basan en pruebas débiles, por lo que son las menos fiables.

La principal desventaja es que al no existir sub categorías en algunas situaciones es difícil entregar un GR; por ejemplo, en el caso de un ensayo clínico (EC) con una muestra pequeña y riesgo de sesgo moderado, el GR ¿sería A o B? La otra desventaja que presenta es que dependen fundamentalmente de diseños clásicos y robustos, y no considera estudios menos habituales o rigurosos (por ejemplo estudios de corte transversal, poblacionales, etc.). Sin embargo, esta clasificación fue pionera y ha servido de base para el desarrollo de clasificaciones más completas, como la propuesta del Centre for EvidenceBased Medicine (CEBM) y otras.

\begin{tabular}{|l|l|}
\hline Validez interna & Interpretación \\
\hline Buena & $\begin{array}{l}\text { Un estudio (incluido RS y meta-análisis) que cumple los criterios específicos de un } \\
\text { estudio bien diseñado. }\end{array}$ \\
\hline Moderada & $\begin{array}{l}\text { Un estudio (incluido RS y meta-análisis) que no cumple (o no está claro que cum- } \\
\text { pla) al menos uno de los criterios específicos de un estudio bien diseñado, aunque } \\
\text { no tenga defectos metodológicos graves. }\end{array}$ \\
\hline Insuficiente & $\begin{array}{l}\text { Un estudio (incluido RS y meta-análisis) que tiene en su diseño al menos un defecto } \\
\text { metodológico grave, o que no cumple (o no está claro que cumpla), al menos uno } \\
\text { de los criterios específicos de un estudio bien diseñado. O, que no tenga defectos } \\
\text { metodológicos graves, pero que acumule defectos menores que hagan que los } \\
\text { resultados del estudio no permitan plantear recomendaciones. }\end{array}$ \\
\hline
\end{tabular}

Figura 3. Validez interna e interpretación de los tipos de estudio para intervenciones de prevención (CTFPHC).

\section{U.S. Preventive Services Task Force}

El U.S. Preventive Services Task Force (USPSTF) es un grupo independiente de expertos en prevención y MBE, creado en 1984 en EE.UU. Es el encargado de valorar de forma rigurosa la investigación clínica con el fin de evaluar los méritos de las medidas preventivas, incluidas las pruebas de detección, servicios de asesoramiento, vacunas y medicamentos preventivos ${ }^{12}$.

Generaron una jerarquización, estableciendo la fuerza de sus recomendaciones a partir de la calidad de la evidencia y del beneficio neto, el que fue definido como beneficio menos daño del servicio preventivo, evaluado tal como se aplica en la atención primaria a la población general.

El USPSTF asigna un nivel de certeza para evaluar el beneficio neto de un servicio preventivo basado en la naturaleza de la evidencia total disponible para sustentar el GR (Figura 5).

\begin{tabular}{|c|c|c|c|c|c|}
\hline GR & NE & $\begin{array}{l}\text { Terapia, prevención, etiología y } \\
\text { daño }\end{array}$ & Pronóstico & Diagnóstico & Estudios económicos \\
\hline \multirow[t]{2}{*}{ A } & $1 \mathrm{a}$ & RS de EC con AA & $\begin{array}{l}\text { RS con homogeneidad y Meta-análisis } \\
\text { de estudios de cohortes concurrentes }\end{array}$ & RS de estudios de diagnóstico nivel 1 & RS de estudios económicos nivel 1 \\
\hline & $1 \mathrm{~b}$ & $\begin{array}{l}\text { EC con AA e intervalo de confianza } \\
\text { estrecho }\end{array}$ & $\begin{array}{l}\text { Estudio individual de cohortes concu- } \\
\text { rrente con seguimiento superior del } \\
80 \% \text { de la cohorte }\end{array}$ & $\begin{array}{l}\text { Comparación independiente y enmas- } \\
\text { carada de un espectro de pacientes } \\
\text { consecutivos, sometidos a la prueba } \\
\text { diagnóstica y al estándar de referencia }\end{array}$ & $\begin{array}{l}\text { Análisis que compara los desenlaces } \\
\text { posibles contra una medida de costos. } \\
\text { Incluye un análisis de sensibilidad }\end{array}$ \\
\hline \multirow[t]{4}{*}{ B } & $2 a$ & RS de estudios de cohortes & RS de estudios de cohortes históricas & $\begin{array}{l}\text { RS de estudios de diagnósticos de nivel } \\
\text { mayor que } 1\end{array}$ & $\begin{array}{l}\text { RS de estudios económicos de nivel } \\
\text { mayor que } 1\end{array}$ \\
\hline & $2 b$ & $\begin{array}{l}\text { Estudios de cohortes individuales. EC } \\
\text { de baja calidad }\end{array}$ & $\begin{array}{l}\text { Estudio individual de cohortes his- } \\
\text { tóricas }\end{array}$ & $\begin{array}{l}\text { Comparación independiente y enmas- } \\
\text { carada de pacientes no consecutivos, } \\
\text { sometidos a la prueba diagnóstica y al } \\
\text { estándar de referencia }\end{array}$ & $\begin{array}{l}\text { Comparación de un número limitado } \\
\text { de desenlaces contra una medida de } \\
\text { costo. Incluye análisis de sensibilidad }\end{array}$ \\
\hline & $3 a$ & $\begin{array}{l}\text { RS con homogeneidad de estudios de } \\
\text { casos y controles }\end{array}$ & & & \\
\hline & $3 b$ & $\begin{array}{l}\text { Estudio de casos y controles indivi- } \\
\text { duales }\end{array}$ & & $\begin{array}{l}\text { Estudios no consecutivos o carentes de } \\
\text { un estándar de referencia }\end{array}$ & $\begin{array}{l}\text { Análisis sin una medida exacta de cos- } \\
\text { to, con análisis de sensibilidad }\end{array}$ \\
\hline $\mathrm{C}$ & 4 & $\begin{array}{l}\text { Series de casos. Estudios de cohortes } \\
\text { y de casos y controles de mala calidad }\end{array}$ & $\begin{array}{l}\text { Series de casos. Estudios de cohortes } \\
\text { de mala calidad }\end{array}$ & $\begin{array}{l}\text { Estudios de casos y controles sin la } \\
\text { aplicación de un estándar de refe- } \\
\text { rencia }\end{array}$ & Estudio sin análisis de sensibilidad \\
\hline D & 5 & $\begin{array}{l}\text { Opinión de expertos sin evaluación } \\
\text { crítica explícita, o basada en fisiología, } \\
\text { o en investigación teórica }\end{array}$ & $\begin{array}{l}\text { Opinión de expertos sin evaluación } \\
\text { crítica explícita, o basada en fisiología, } \\
\text { o en investigación teórica }\end{array}$ & $\begin{array}{l}\text { Opinión de expertos sin evaluación } \\
\text { crítica explícita, o basada en fisiología, } \\
\text { o en investigación teórica }\end{array}$ & $\begin{array}{l}\text { Opinión de expertos sin evaluación } \\
\text { crítica explícita, o basada en investiga- } \\
\text { ción económica }\end{array}$ \\
\hline
\end{tabular}

Figura 4. Clasificación de los niveles de evidencia según Sackett. 
Figura 5. Descripción de los niveles de evidencia para exámenes periódicos de salud (USPSTF)

Figura 6. Grados de recomendación para exámenes periódicos de salud (USPSTF).

\begin{tabular}{|l|l|}
\hline $\begin{array}{c}\text { Niveles de } \\
\text { certeza }\end{array}$ & Descripción \\
\hline Alta & $\begin{array}{l}\text { La evidencia disponible incluye resultados consistentes de estudios bien diseñados, bien conducidos en poblaciones represen- } \\
\text { tativas de atención primaria. Estos estudios evalúan los efectos del servicio de prevención en la salud. Esta conclusión es por lo } \\
\text { tanto poco probable que sea fuertemente afectada por los resultados de futuros estudios }\end{array}$ \\
\hline Moderada & $\begin{array}{l}\text { La evidencia disponible no es suficiente para determinar los efectos de la acción preventiva, pero la confianza en la estimación } \\
\text { se ve limitada por factores tales como: } \\
\text { - Número, tamaño o calidad de los estudios individuales } \\
\text { - Inconsistencia de los resultados entre los estudios individuales } \\
\text { - Generalización limitada de los resultados en la práctica habitual la atención primaria } \\
\text { - Falta de coherencia en la cadena de la evidencia existente } \\
\text { A medida que más información se encuentre disponible, la magnitud o la dirección del efecto observado podría cambiar, y este } \\
\text { cambio puede ser lo suficientemente importante como para alterar la conclusión }\end{array}$ \\
\hline $\begin{array}{l}\text { Baja } \\
\text { - Limitencia disponible es insuficiente para evaluar los efectos sobre los resultados de salud, debido a: } \\
\text { - Defectos importantes en el diseño del estudio o los métodos } \\
\text { - Inconsistencia de los resultados entre los estudios individuales } \\
\text { - Lagunas en la cadena de la evidencia existente } \\
\text { - Hallazgos no generalizables para la práctica habitual de la atención primaria } \\
\text { - Falta de información sobre los resultados importantes de salud } \\
\text { Más información puede permitir la estimación de los efectos sobre los resultados de salud }\end{array}$ \\
\hline
\end{tabular}

\begin{tabular}{|c|c|c|}
\hline $\begin{array}{l}\text { Recomen- } \\
\text { dación }\end{array}$ & Interpretación & Sugerencia para la práctica \\
\hline A & $\begin{array}{l}\text { Se recomienda la acción preventiva. Existe alta certeza que el bene- } \\
\text { ficio neto es substancial }\end{array}$ & Ofrecer o proporcionar este servicio \\
\hline B & $\begin{array}{l}\text { Se recomienda la acción preventiva. Hay una alta certeza de que el } \\
\text { beneficio neto es moderado o existe moderada certeza de que el } \\
\text { beneficio neto es de moderado a sustancial }\end{array}$ & Ofrecer o proporcionar este servicio \\
\hline C & $\begin{array}{l}\text { Se recomienda selectivamente el ofrecimiento o la prestación de } \\
\text { este servicio a los pacientes individuales basadas en criterios profe- } \\
\text { sionales y las preferencias del paciente. Hay por lo menos moderada } \\
\text { certeza que el beneficio neto es pequeño }\end{array}$ & $\begin{array}{l}\text { Ofrecer o proporcionar este servicio para los pacientes } \\
\text { seleccionados en función de las circunstancias indivi- } \\
\text { duales }\end{array}$ \\
\hline D & $\begin{array}{l}\text { NO se recomienda la acción preventiva. Hay certeza moderada o } \\
\text { alta que el servicio no tiene ningún beneficio neto o que los daños } \\
\text { son mayores que los beneficios }\end{array}$ & Desalentar el uso de este servicio \\
\hline I & $\begin{array}{l}\text { Se concluye que la evidencia actual es insuficiente para evaluar el } \\
\text { equilibrio entre los beneficios y los daños de la acción preventiva. } \\
\text { La evidencia es deficiente, de mala calidad, o es contradictoria, y el } \\
\text { balance de riesgos y beneficios no se puede determinar }\end{array}$ & $\begin{array}{l}\text { Lea la sección de consideraciones clínicas de las reco- } \\
\text { mendaciones de la USPSTF. Si el servicio es ofrecido, los } \\
\text { pacientes deben comprender la incertidumbre que existe } \\
\text { sobre el equilibrio entre beneficios y daños }\end{array}$ \\
\hline
\end{tabular}

A los GR se le asigna una letra (A, B, C, D, o I). Se apoyan en grados de certeza, que se definen como la probabilidad que el beneficio neto de un servicio preventivo que ha sido evaluado por la USPSTF sea correcto. El grado $\mathrm{A}$, sugiere recomendar la acción ya que existe un alto grado de certeza que el beneficio neto es sustancial; el extremo opuesto es el grado I, que sugiere que no hay suficiente evidencia para evaluar el beneficio neto de una acción y por lo tanto, no se puede recomendar.

El USPSTF ha actualizado sus definiciones de las calificaciones que asigna a las recomendaciones y ahora incluye una columna de "sugerencias para la práctica" asociados con cada grado (Figura 6) ${ }^{13}$.

\section{Centre for Evidence-Based Medicine, Oxford}

La propuesta del Centre for Evidence-Based Medicine de Oxford (CEBM) se caracteriza por valorar la evidencia según el área temática o escenario clínico y el tipo de estudio que involucra al problema clínico en cuestión ${ }^{14}$. Es una innovación complementaria a otras iniciativas. Tiene la ventaja que gradúa la evidencia de acuerdo al mejor diseño para cada escenario clínico, otorgándole intencionalidad, agregando las revisiones sistemáticas (RS) en los distintos ámbitos. Por ejemplo, al tratarse de un escenario de pronóstico, la evidencia será valorada a partir de una RS de estudios de cohortes con homogeneidad, o en su defecto, de estudios de cohortes individuales con un seguimiento superior al $80 \%$ de la cohorte; en cambio, si el escenario se refiere a terapia o tratamiento, la evidencia se valorará principalmente a partir de RS de EC, o en su defecto de EC individuales con intervalos de confianza estrechos.

Esta clasificación tiene la ventaja que nos asegura el conocimiento más atingente a cada escenario, por su alto grado de especialización. Además tiene la prerrogativa de aclarar cómo afecta la falta de rigurosidad metodológica al diseño de los estudios, disminuyendo su valoración no sólo en la gradación de la evidencia, sino que también en la fuerza de las recomendaciones (Figura 7). 
No obstante lo cual, presenta algunos inconvenientes para su práctica habitual. Por una parte, vemos como en su estructura se presentan términos epidemiológicos poco amigables y con múltiples aclaraciones que hacen su lectura poco fluida $\mathrm{y}$, que rápidamente pueden frustrar a quien se aproxima a ella por primera vez. En su intento por abarcar todos los aspectos con la máxima exhaustividad, pierde la simpleza para hacerla aplicable ${ }^{6}$.

El año 2009 se realizó una revisión de la tabla original con la idea de simplificarla para permitir una búsqueda heurística rápida y al mismo tiempo que permitiese jerarquizar la evidencia encontrada. Se adicionó un

\begin{tabular}{|c|c|c|c|c|c|c|}
\hline GR & NE & $\begin{array}{l}\text { Tratamiento, prevención, } \\
\text { etiología y daño }\end{array}$ & $\begin{array}{l}\text { Pronóstico e historia } \\
\text { natural }\end{array}$ & Diagnóstico & $\begin{array}{l}\text { Diagnóstico diferencial y } \\
\text { prevalencia }\end{array}$ & $\begin{array}{l}\text { Estudios económicos y de } \\
\text { análisis de decisión }\end{array}$ \\
\hline \multirow[t]{3}{*}{ A } & $1 \mathrm{a}$ & $\begin{array}{l}\text { RS con homogeneidad de EC } \\
\text { con asignación aleatoria }\end{array}$ & $\begin{array}{l}\text { RS de estudios de cohortes } \\
\text { con homogeneidad (que in- } \\
\text { cluya estudios con resultados } \\
\text { comparables, en la misma } \\
\text { dirección y validados en dife- } \\
\text { rentes poblaciones) }\end{array}$ & $\begin{array}{l}\text { RS de estudios de diagnóstico } \\
\text { de alta calidad con homoge- } \\
\text { neidad (que incluya estudios } \\
\text { con resultados comparables, } \\
\text { en la misma dirección y en } \\
\text { diferentes centros clínicos) }\end{array}$ & $\begin{array}{l}\text { RS con homogeneidad de } \\
\text { estudios de cohortes pros- } \\
\text { pectivas }\end{array}$ & $\begin{array}{l}\text { RS con homogeneidad de } \\
\text { estudios económicos de alta } \\
\text { calidad }\end{array}$ \\
\hline & $1 b$ & $\begin{array}{l}\text { EC individual con intervalo de } \\
\text { confianza estrecho }\end{array}$ & $\begin{array}{l}\text { Estudios de cohortes indivi- } \\
\text { duales, con un seguimiento } \\
\text { mayor de } 80 \% \text { de las cohor- } \\
\text { tes y validadas en una sola } \\
\text { población }\end{array}$ & $\begin{array}{l}\text { Estudios de cohortes que vali- } \\
\text { den la calidad de una prueba } \\
\text { específica, con estándar de } \\
\text { referencia adecuado o a partir } \\
\text { de algoritmos de estimación } \\
\text { del pronóstico o de catego- } \\
\text { rización del diagnóstico o } \\
\text { probado en un centro clínico }\end{array}$ & $\begin{array}{l}\text { Estudios de cohortes prospec- } \\
\text { tivas con buen seguimiento }\end{array}$ & $\begin{array}{l}\text { Análisis basado en costes } \\
\text { o alternativas clínicamente } \\
\text { sensibles; RS de la evidencia. } \\
\text { Incluye análisis de sensibilidad }\end{array}$ \\
\hline & 1c & Todos o ninguna & $\begin{array}{l}\text { Series de casos (todos o } \\
\text { ninguno) }\end{array}$ & $\begin{array}{l}\text { Pruebas diagnósticas con } \\
\text { especificidad tan alta que un } \\
\text { resultado positivo confirma el } \\
\text { diagnóstico y con sensibilidad } \\
\text { tan alta que un resultado ne- } \\
\text { gativo descarta el diagnóstico }\end{array}$ & $\begin{array}{l}\text { Series de casos (todos o } \\
\text { ninguno) }\end{array}$ & $\begin{array}{l}\text { Análisis en términos absolutos } \\
\text { de riesgos y } \\
\text { beneficios clínicos: claramente } \\
\text { tan buenas o } \\
\text { mejores, pero más baratas, } \\
\text { claramente tan malas o peores } \\
\text { pero más caras }\end{array}$ \\
\hline \multirow[t]{5}{*}{ B } & $2 a$ & $\begin{array}{l}\text { RS de estudios de cohortes } \\
\text { con homogeneidad }\end{array}$ & $\begin{array}{l}\text { RS de estudios de cohortes } \\
\text { históricas o de grupos con- } \\
\text { troles no tratados en EC con } \\
\text { homogeneidad }\end{array}$ & $\begin{array}{l}\text { RS de estudios de diagnós- } \\
\text { ticos de nivel } 2 \text { con homo- } \\
\text { geneidad }\end{array}$ & $\begin{array}{l}\text { RS con homogeneidad de } \\
\text { estudios } 2 \text { b y mejores }\end{array}$ & $\begin{array}{l}\text { RS con homogeneidad de } \\
\text { estudios económicos con nivel } \\
\text { mayor a } 2\end{array}$ \\
\hline & $2 b$ & $\begin{array}{l}\text { Estudios de cohortes indivi- } \\
\text { duales con seguimiento infe- } \\
\text { rior a } 80 \% \text {. EC de baja calidad }\end{array}$ & $\begin{array}{l}\text { Estudio individual de cohortes } \\
\text { históricas o seguimiento de } \\
\text { controles no tratados en un } \\
\text { EC o guía de práctica clínica } \\
\text { no validada }\end{array}$ & $\begin{array}{l}\text { Estudios exploratorios que } \\
\text { a través de una regresión } \\
\text { logística determinan factores } \\
\text { significativos y validados con } \\
\text { estándar de referencia ade- } \\
\text { cuado (independiente de la } \\
\text { prueba diagnóstica) }\end{array}$ & $\begin{array}{l}\text { Estudio individual de cohortes } \\
\text { históricas o de seguimiento } \\
\text { insuficiente }\end{array}$ & $\begin{array}{l}\text { Análisis basado en costes o } \\
\text { alternativas clínicamente sen- } \\
\text { sibles; limitado a revisión de la } \\
\text { evidencia. Incluye análisis de } \\
\text { sensibilidad }\end{array}$ \\
\hline & $2 c$ & $\begin{array}{l}\text { Estudios ecológicos o de resul- } \\
\text { tados en salud }\end{array}$ & $\begin{array}{l}\text { Investigación de resultados } \\
\text { en salud }\end{array}$ & & Estudios ecológicos & $\begin{array}{l}\text { Auditorías o estudios de resul- } \\
\text { tados en salud }\end{array}$ \\
\hline & $3 a$ & $\begin{array}{l}\text { RS de estudios de casos y } \\
\text { controles con homogeneidad }\end{array}$ & & $\begin{array}{l}\text { RS de estudios con homoge- } \\
\text { neidad de estudios } 3 \text { b y mejor } \\
\text { calidad }\end{array}$ & $\begin{array}{l}\text { RS de estudios con homoge- } \\
\text { neidad de estudios } 3 \text { b y mejor } \\
\text { calidad }\end{array}$ & $\begin{array}{l}\text { RS de estudios con homoge- } \\
\text { neidad de estudios } 3 \text { b y mejor } \\
\text { calidad }\end{array}$ \\
\hline & $3 b$ & $\begin{array}{l}\text { Estudios de casos y controles } \\
\text { individuales }\end{array}$ & & $\begin{array}{l}\text { Comparación enmascarada } \\
\text { y objetiva de un espectro de } \\
\text { pacientes que podría ser exa- } \\
\text { minado para un determinado } \\
\text { trastorno, pero el estándar de } \\
\text { referencia no se aplica a todos } \\
\text { los pacientes del estudio. Es- } \\
\text { tudios no consecutivos o sin } \\
\text { aplicación de un estándar de } \\
\text { referencia }\end{array}$ & & $\begin{array}{l}\text { Estudio no consecutivo de co- } \\
\text { horte, o análisis muy limitado } \\
\text { de la población basado en } \\
\text { pocas alternativas o costes, } \\
\text { datos de mala calidad, pero } \\
\text { con análisis de sensibilidad } \\
\text { que incorporan variaciones } \\
\text { clínicamente sensibles }\end{array}$ \\
\hline C & 4 & $\begin{array}{l}\text { Series de casos, estudios de } \\
\text { cohortes y de casos y contro- } \\
\text { les de baja calidad }\end{array}$ & $\begin{array}{l}\text { Series de casos y estudios de } \\
\text { cohortes de pronóstico de } \\
\text { baja calidad }\end{array}$ & $\begin{array}{l}\text { Estudios de casos y controles } \\
\text { con escasos o sin estándares } \\
\text { de referencia independientes }\end{array}$ & $\begin{array}{l}\text { Series de casos o estándares } \\
\text { de referencia obsoletos }\end{array}$ & $\begin{array}{l}\text { Análisis sin análisis de sen- } \\
\text { sibilidad }\end{array}$ \\
\hline D & 5 & $\begin{array}{l}\text { Opinión de expertos sin eva- } \\
\text { luación crítica explícita, ni ba- } \\
\text { sada en fisiología, ni en traba- } \\
\text { jo de investigación juicioso, ni } \\
\text { en "principios fundamentales" }\end{array}$ & $\begin{array}{l}\text { Opinión de expertos sin eva- } \\
\text { luación crítica explícita, ni ba- } \\
\text { sada en fisiología, ni en traba- } \\
\text { jo de investigación juicioso, ni } \\
\text { en "principios fundamentales" }\end{array}$ & $\begin{array}{l}\text { Opinión de expertos sin eva- } \\
\text { luación crítica explícita, ni ba- } \\
\text { sada en fisiología, ni en traba- } \\
\text { jo de investigación juicioso, ni } \\
\text { en "principios fundamentales" }\end{array}$ & $\begin{array}{l}\text { Opinión de expertos sin eva- } \\
\text { luación crítica explícita, ni ba- } \\
\text { sada en fisiología, ni en traba- } \\
\text { jo de investigación juicioso, ni } \\
\text { en "principios fundamentales" }\end{array}$ & $\begin{array}{l}\text { Opinión de expertos sin eva- } \\
\text { luación crítica explícita, ni ba- } \\
\text { sada en fisiología, ni en traba- } \\
\text { jo de investigación juicioso, ni } \\
\text { en "principios fundamentales" }\end{array}$ \\
\hline
\end{tabular}

Figura 7. Niveles de evidencia de CEBM (2009). 


\begin{tabular}{|c|c|c|c|c|c|}
\hline Pregunta & Paso 1 (Nivel $1^{*}$ ) & Paso 2 (Nivel $2^{*}$ ) & Paso 3 (Nivel $3^{*}$ ) & Paso 4 (Nivel $4^{*}$ ) & Paso 5 (Nivel $5^{*}$ ) \\
\hline $\begin{array}{l}\text { ¿Qué tan común es el } \\
\text { problema? }\end{array}$ & $\begin{array}{l}\text { Encuestas locales y actua- } \\
\text { les aleatorias de la mues- } \\
\text { tra (o censos) }\end{array}$ & $\begin{array}{l}\text { RS de encuestas que } \\
\text { coincidan con las circuns- } \\
\text { tancias locales }\end{array}$ & Muestra local no aleatoria & Serie de casos & N/A \\
\hline $\begin{array}{l}\text { ¿Es preciso el test } \\
\text { de monitoreo o test } \\
\text { diagnóstico? } \\
\text { (Diagnóstico) }\end{array}$ & $\begin{array}{l}\text { RS de estudios transver- } \\
\text { sales con estándar de } \\
\text { referencia aplicado de } \\
\text { forma consistente y con } \\
\text { enmascaramiento }\end{array}$ & $\begin{array}{l}\text { Estudios individuales de } \\
\text { corte transversal con es- } \\
\text { tándar de referencia apli- } \\
\text { cado de forma consistente } \\
\text { y con enmascaramiento }\end{array}$ & $\begin{array}{l}\text { Estudios no consecutivos, } \\
\text { estudios sin un estándar } \\
\text { de referencia aplicado de } \\
\text { forma consistente }\end{array}$ & $\begin{array}{l}\text { Estudios caso control o es- } \\
\text { tándar de referencia pobre } \\
\text { o no independiente }\end{array}$ & $\begin{array}{l}\text { Mecanismos basados en el } \\
\text { razonamiento }\end{array}$ \\
\hline $\begin{array}{l}\text { ¿Qué pasaría si no se } \\
\text { agrega una terapia? } \\
\text { (Pronóstico) }\end{array}$ & $\begin{array}{l}\text { RS de estudios de cohorte } \\
\text { de inicio }\end{array}$ & $\begin{array}{l}\text { Estudios de cohorte de } \\
\text { inicio }\end{array}$ & $\begin{array}{l}\text { Estudio de cohorte o el } \\
\text { brazo control de un EC } \\
\text { con AA * }\end{array}$ & $\begin{array}{l}\text { Estudio de caso-control o } \\
\text { estudios de cohorte pro- } \\
\text { nóstica de pobre calidad }\end{array}$ & $\mathrm{N} / \mathrm{A}$ \\
\hline $\begin{array}{l}\text { ¿Esta intervención ayuda? } \\
\text { (beneficios del } \\
\text { tratamiento) }\end{array}$ & $\begin{array}{l}\text { RS de EC con AA o ensa- } \\
\text { yos n-de- } 1\end{array}$ & $\begin{array}{l}\text { EC con AA o estudios } \\
\text { observacionales con un } \\
\text { efecto dramático }\end{array}$ & $\begin{array}{l}\text { Estudio de cohorte, con } \\
\text { seguimiento controlado } \\
\sin A A^{*}\end{array}$ & $\begin{array}{l}\text { Serie de casos, estudios } \\
\text { caso-control o estudios } \\
\text { históricos controlados }\end{array}$ & $\begin{array}{l}\text { Mecanismos basados en el } \\
\text { razonamiento }\end{array}$ \\
\hline $\begin{array}{l}\text { ¿Cuáles son los daños } \\
\text { comunes? } \\
\text { (efectos nocivos del } \\
\text { tratamiento) }\end{array}$ & $\begin{array}{l}\text { RS de EC con AA, RS de } \\
\text { estudios de casos y con- } \\
\text { troles anidados, ensayo } \\
\text { n-de-1 con el paciente } \\
\text { sobre el que está plan- } \\
\text { teando la pregunta, o } \\
\text { estudio observacional con } \\
\text { un efecto dramático. }\end{array}$ & $\begin{array}{l}\text { EC individual con AA o } \\
\text { (excepcionalmente) estu- } \\
\text { dios observacionales con } \\
\text { un efecto dramático }\end{array}$ & $\begin{array}{l}\text { Estudio de cohorte, con } \\
\text { seguimiento controlado } \\
\text { (post-comercialización) } \\
\text { con un número suficiente } \\
\text { para descartar un daño } \\
\text { común. (Para los daños a } \\
\text { largo plazo, la duración } \\
\text { del seguimiento debe ser } \\
\text { suficiente) }\end{array}$ & $\begin{array}{l}\text { Series de casos, estudios } \\
\text { de casos y controles, o } \\
\text { estudios históricos con- } \\
\text { trolados }\end{array}$ & $\begin{array}{l}\text { Mecanismos basados en el } \\
\text { razonamiento }\end{array}$ \\
\hline $\begin{array}{l}\text { ¿Cuáles son los daños } \\
\text { raros? } \\
\text { (efectos nocivos del } \\
\text { tratamiento) }\end{array}$ & $\begin{array}{l}\text { RS de EC con AA ó ensayo } \\
n-d e-1\end{array}$ & $\begin{array}{l}\text { EC con AA o (excepcio- } \\
\text { nalmente) estudios obser- } \\
\text { vacionales con un efecto } \\
\text { dramático }\end{array}$ & & & \\
\hline $\begin{array}{l}\text { ¿Vale la pena esta prueba } \\
\text { para detección temprana? } \\
\text { (tamizaje) }\end{array}$ & RS de EC con AA & $E C \operatorname{con} A A$ & $\begin{array}{l}\text { Estudios de cohortes con } \\
\text { seguimiento controlado }\end{array}$ & $\begin{array}{l}\text { Serie de casos, estudios de } \\
\text { casos y controles o estu- } \\
\text { dios históricos controlados }\end{array}$ & $\begin{array}{l}\text { Mecanismos basados en el } \\
\text { razonamiento }\end{array}$ \\
\hline
\end{tabular}

Figura 8. Niveles de evidencia de CEBM (2011).

glosario con la definición de los términos relevantes, en forma precisa y entendible. Además, se consideró que las pruebas de "screening" o tamizaje debían considerarse como una entrada independiente y que se debía resaltar la importancia de las RS.

En esta nueva versión ${ }^{15}$, se distinguen las filas que representan la serie de pasos que se deberían seguir en búsqueda de la mejor evidencia. Hacia el extremo izquierdo se encuentra la evidencia más fuerte y hacia la derecha la más débil. Las columnas representan los tipos de preguntas que el clínico pudiera encontrarse: ¿Qué tan común es el problema?, ¿Es precisa la prueba diagnóstica?, ¿Qué pasaría si no se instaura el tratamiento?, ¿La intervención ayudará al paciente?, ¿Qué tan frecuente o infrecuente es la complicación?, ¿Vale la pena utilizar esta prueba diagnóstica para detección precoz? (Figura 8).

En primer lugar se debe identificar a cuál de las preguntas de la columna izquierda corresponde el artículo del que proviene la evidencia que se desea valorar. Posteriormente, se debe identificar en la fila hacia la derecha, el diseño del estudio. Este punto es el que indica el NE, el que se identifica en la fila superior. Si bien la tabla es de fácil manejo y permite una valoración rápida y práctica de la evidencia, presenta al mismo tiempo la desventaja que al no existir subcategorías en relación a la calidad de los estudios, puede sesgar el valor que se le otorga a la evidencia. Por ejemplo, si la evidencia encontrada para una pregunta de terapia proviene de una RS, probablemente se categorizará como NE 1, pero si al continuar con la búsqueda nos encontramos con un EC con doble enmascaramiento y alta calidad metodológica que presenta un resultado contrario a la RS, ¿a cuál le creeremos? No sólo el NE que representa un determinado diseño va a avalar la decisión ya que pueden existir RS con fallas metodológicas que limitan la validez del estudio, situaciones en que las subcategorías serían de gran ayuda; sin embargo, al incluir estas subcategorías la simpleza de esta herramienta se pierde. Por lo tanto, CEBM sugiere que los NE se interpreten con una dosis de sentido común y buen juicio, lo que es posible lograr realizando una sistemática y exhaustiva búsqueda de la literatura científica que permita obtener los artículos relevantes y realizar un acucioso análisis crítico de la literatura antes de valorar la evidencia y aplicarla en la práctica si así lo amerita ${ }^{16}$.

No obstante que CEBM actualizó la propuesta, es menester señalar que la anterior (Figura 7), sigue siendo utilizando de forma masiva por los grupos de investigación, que ven en ésta una mejora de la clasificación de Sackett. 


\begin{tabular}{|c|c|c|c|}
\hline $\begin{array}{l}\text { Grado de recomendación. } \\
\text { Descripción. }\end{array}$ & Beneficio vs. Riesgo y cargas & $\begin{array}{l}\text { Calidad metodológica que apoya la } \\
\text { evidencia }\end{array}$ & Implicancias \\
\hline $\begin{array}{l}\text { 1A. Recomendación fuerte, } \\
\text { evidencia de alta calidad }\end{array}$ & $\begin{array}{l}\text { Los beneficios superan claramente los riesgos } \\
\text { y cargas, o viceversa. }\end{array}$ & $\begin{array}{l}\text { EC sin importantes limitaciones o evidencia } \\
\text { abrumadora de estudios observacionales. }\end{array}$ & $\begin{array}{l}\text { Recomendación fuerte, puede aplicarse a la } \\
\text { mayoría de los pacientes en la mayoría de } \\
\text { circunstancias, sin reserva. }\end{array}$ \\
\hline $\begin{array}{l}\text { 1B. Recomendación fuerte, } \\
\text { evidencia de moderada calidad }\end{array}$ & $\begin{array}{l}\text { Los beneficios superan claramente los riesgos } \\
\text { y cargas, o viceversa. }\end{array}$ & $\begin{array}{l}\text { EC con importantes limitaciones (resultados } \\
\text { inconsistentes, defectos metodológicos, } \\
\text { indirectos o imprecisos) o pruebas excep- } \\
\text { cionalmente fuertes a partir de estudios } \\
\text { observacionales. }\end{array}$ & $\begin{array}{l}\text { Recomendación fuerte, puede aplicarse a la } \\
\text { mayoría de los pacientes en la mayoría de } \\
\text { circunstancias, sin reserva }\end{array}$ \\
\hline $\begin{array}{l}\text { 1C. Recomendación fuerte, } \\
\text { evidencia de baja o muy baja } \\
\text { calidad }\end{array}$ & $\begin{array}{l}\text { Los beneficios superan claramente los riesgos } \\
\text { y cargas, o viceversa. }\end{array}$ & Estudios observacionales o series de casos. & $\begin{array}{l}\text { Recomendación fuerte, pero puede cambiar } \\
\text { cuando se disponga de mayor evidencia de } \\
\text { calidad. }\end{array}$ \\
\hline $\begin{array}{l}\text { 2A. Recomendación débil, } \\
\text { evidencia de alta calidad }\end{array}$ & $\begin{array}{l}\text { Beneficios estrechamente equilibrados con los } \\
\text { riesgos y la carga. }\end{array}$ & $\begin{array}{l}\text { EC sin importantes limitaciones o evidencia } \\
\text { abrumadora de estudios observacionales. }\end{array}$ & $\begin{array}{l}\text { Recomendación débil, la mejor acción puede } \\
\text { variar dependiendo de las circunstancias de } \\
\text { los pacientes o de los valores de la sociedad. }\end{array}$ \\
\hline $\begin{array}{l}\text { 2B. Recomendación débil, } \\
\text { evidencia de moderada calidad }\end{array}$ & $\begin{array}{l}\text { Beneficios estrechamente equilibrados con los } \\
\text { riesgos y la carga. }\end{array}$ & $\begin{array}{l}\text { EC con importantes limitaciones (resultados } \\
\text { inconsistentes, defectos metodológicos, } \\
\text { indirectos o imprecisos) o pruebas excep- } \\
\text { cionalmente fuertes a partir de estudios } \\
\text { observacionales. }\end{array}$ & $\begin{array}{l}\text { Recomendación débil, la mejor acción puede } \\
\text { variar dependiendo de las circunstancias de } \\
\text { los pacientes o de los valores de la sociedad. }\end{array}$ \\
\hline $\begin{array}{l}\text { 2C. Recomendación débil, } \\
\text { evidencia de baja o muy baja } \\
\text { calidad }\end{array}$ & $\begin{array}{l}\text { Incertidumbre en las estimaciones de be- } \\
\text { neficios, riesgos y cargas; los beneficios, } \\
\text { riesgos, y la carga puede estar estrechamente } \\
\text { equilibrado. }\end{array}$ & Estudios observacionales o series de casos. & $\begin{array}{l}\text { Recomendaciones muy débiles, otras alter- } \\
\text { nativas pueden ser igualmente razonables. }\end{array}$ \\
\hline
\end{tabular}

Figura 9. GRADE modificado: grados de recomendación.

\section{Grade Working Group}

GRADE (The Grading of Recommendations Assessment, Development and Evaluation), es un sistema para clasificar la calidad de la evidencia y fuerza de recomendación aplicable a una amplia gama de intervenciones y contextos. Fue elaborado en base a la experiencia previa con otras herramientas existentes para conseguir un sistema "más razonable, confiable y ampliamente aplicable"17.

La principal diferencia de este sistema en relación a otros, es que GRADE no valora la calidad de un estudio individual; sino que le da un valor a la evidencia para una medida resultado en particular, a partir de varios estudios primarios.

Los juicios sobre la fuerza de una recomendación deben tener en cuenta el balance entre beneficios y riesgos, la calidad de la evidencia, la aplicación de ésta en circunstancias específicas y la situación de riesgo basal, que son los puntos claves evaluados en cada artículo (Figura 9).

Destaca en esta propuesta, la elaboración de una tabla de síntesis que se obtiene de forma sistemática y que se basa en la evaluación de la calidad de la evidencia según el tipo de diseño ${ }^{18}$ : EC con asignación aleatoria: calidad alta; estudios observacionales: calidad baja; y cualquier otra evidencia: calidad muy baja.

El grado disminuye si es que en la calidad del estudio existe: una limitación importante (-1) o muy importante (-2); una inconsistencia importante (-1); incertidumbre respecto de si la evidencia es directa o indirecta: si es alguna (-1) y si es máxima (-2); información imprecisa o escasa (-1); y alta probabilidad de sesgo de información (-1).

Por otra parte, el grado aumenta si es que: la evidencia de la asociación es fuerte, con un $\mathrm{RR}>$ a 2 ó $<0,5$ basado en evidencia consistente derivada de dos o más estudios observacionales, sin factores de confusión plausibles $(+1)$; o cuando la evidencia de la asociación es muy fuerte, con un $\mathrm{RR}>$ a 5 ó $<0,2$ basado en evidencia directa, sin amenazas importantes para la validez $(+2)$; o cuando existe evidencia de un gradiente dosis respuesta $(+1)$; o cuando todos los potenciales factores de confusión posibles se han podido controlar en su efecto $(+1)$.

Las ventajas del sistema GRADE respecto de los otros sistemas de clasificación son: que utiliza definiciones explícitas y juicios secuenciales durante el proceso de clasificación; que proporciona una descripción detallada de los criterios para la calidad de la evidencia para los resultados individuales y para la calidad general de la evidencia; que pesa la importancia relativa de los resultados; que considera el equilibrio entre los beneficios de salud versus los daños, costos y gastos; $y$, que permite desarrollar perfiles de evidencia y resúmenes de los hallazgos ${ }^{19}$.

Es una herramienta muy completa que incorpora el uso de un software de uso libre para extraer los datos y realizar la síntesis, por ende, requiere tiempo para su aplicación ya que el análisis de un estudio individual es muy exhaustivo. Ha sido incorporada por diversas instituciones para para evaluar la calidad de la evidencia disponible, realizar recomendaciones y generar guías de práctica clínica. Entre ellas, destacan organismos internacionales tales como World Health Organization, Cochrane Library, World Allergy Organization (WAO), Surviving Sepsis, UpToDate, etc; instituciones norteamericanas como CDC's Healthcare Infection Control Practices 
Advisory Committee (HICPAC), CDC's Division of Viral Hepatitis, Infectious Diseases Society of America, CDC's Advisory Committee on Immunization Practices (ACIP), Agency for Healthcare Research and Quality (AHRQ), The University of Pennsylvania Health System Center for Evidence-based Practice, American College of Physicians, American Thoracic Society, American Gastroenterological Association (AGA), The American Society of Colon and Rectal Surgeons, The Eastern Association for the Surgery of Trauma (EAST), American College of Chest Physicians, American Society for Gastrointestinal Endoscopy (ASGE), The American Association for the Study of Liver Diseases, etc; organizaciones europeas como British Medical Journal (UK), Clinical Evidence (UK), National Institute for Clinical Excellence (NICE, UK), The Scottish Intercollegiate Guidelines Network (SIGN, UK), NHS Quality Improvement (UK), German Center for Evidence-based Nursing "sapere aude" (Alemania), Evidence-based Nursing Südtirol, Alto Adige (Italia), European Society of Thoracic Surgeons, European Respiratory Society, European Association for the Study of the Liver (EASL), Belgian Centre for EvidenceBased Medicine (CEBAM), etc; instituciones canadienses como Health Quality Ontario, The Canadian Agency for Drugs and Technologies in Health, Evidence-Based

\begin{tabular}{|l|l|}
\hline \multicolumn{1}{|c|}{ NE } & Interpretación \\
\hline $\mathbf{1 + +}$ & Meta-análisis de alta calidad, RS de EC ó EC de alta calidad con muy poco riesgo de sesgo \\
\hline $\mathbf{1 +}$ & Meta-análisis bien realizados, RS de EC ó EC bien realizados con poco riesgo de sesgos \\
\hline $\mathbf{1 -}$ & Meta-análisis, RS de EC ó EC con alto riesgo de sesgos \\
\hline $\mathbf{2 + +}$ & $\begin{array}{l}\text { RS de alta calidad de estudios de cohortes o de casos y controles. Estudios de cohortes o } \\
\text { de casos y controles con bajo riesgo de sesgo y con alta probabilidad de establecer una } \\
\text { relación causal }\end{array}$ \\
\hline $\mathbf{2 +}$ & $\begin{array}{l}\text { Estudios de cohortes o de casos y controles bien realizados con bajo riesgo de sesgo y con } \\
\text { una moderada probabilidad de establecer una relación causal }\end{array}$ \\
\hline $\mathbf{2 -}$ & $\begin{array}{l}\text { Estudios de cohortes o de casos y controles con alto riesgo de sesgo y riesgo significativo de } \\
\text { que la relación no sea causal }\end{array}$ \\
\hline $\mathbf{3}$ & Estudios no analíticos, como informes de casos y series de casos \\
\hline $\mathbf{4}$ & Opinión de expertos \\
\hline
\end{tabular}

Figura 10. Niveles de evidencia para estudios de tratamiento. Propuesta del SIGN.

\begin{tabular}{|c|l|}
\hline $\begin{array}{c}\text { Grado de } \\
\text { recomendación }\end{array}$ & Interpretación \\
\hline A & $\begin{array}{l}\text { Al menos un meta-análisis, RS ó EC clasificado como 1++ y directamente } \\
\text { aplicable a la población diana de la guía; o un volumen de evidencia científica } \\
\text { compuesto por estudios clasificados como 1+ y con gran consistencia entre ellos. }\end{array}$ \\
\hline B & $\begin{array}{l}\text { Volumen de evidencia científica compuesta por estudios clasificados como } 2 \\
++, \text { directamente aplicable a la población blanco de la guía y que demuestran } \\
\text { gran consistencia entre ellos; o evidencia científica extrapolada desde estudios } \\
\text { clasificados como 1 ++ ó 1+ }\end{array}$ \\
\hline C & $\begin{array}{l}\text { Volumen de evidencia científica compuesta por estudios clasificados como 2 } \\
+ \text { directamente aplicables a la población blanco de la guía y que demuestran } \\
\text { gran consistencia entre ellos; o evidencia científica extrapolada desde estudios } \\
\text { clasificados como 2 ++ }\end{array}$ \\
\hline D & $\begin{array}{l}\text { Evidencia científica de nivel 3 ó 4; o evidencia científica extrapolada desde estu- } \\
\text { dios clasificados como 2+ }\end{array}$ \\
\hline
\end{tabular}

Figura 11. Grados de recomendación para estudios de tratamiento. Propuesta del SIGN.
Tuberculosis Diagnosis, The Canadian Cardiovascular Society, La Société Canadienne de Cardiologie, Canadian Task Force on Preventive Health Care (CTFPHC), etc. ${ }^{10}$; a las que habría sumar alrededor de 50 instituciones más ${ }^{10}$. En Chile, el Ministerio de Salud, ha hecho interesantes esfuerzos por incorporar GRADE en la elaboración de guías de práctica clínica.

\section{Scottish Intercollegiate Guidelines Network}

El Scottish Intercollegiate Guidelines Network (SIGN) desarrolla guías de práctica clínica basadas en la evidencia, realizadas para el Servicio Nacional de Salud (NHS) de Escocia. Derivan de RS de la literatura científica y son diseñadas como un vehículo para acelerar la traducción del nuevo conocimiento en acción para cumplir con el objetivo de reducir la variabilidad de la práctica y mejorar los resultados relevantes para los pacientes ${ }^{20}$.

La propuesta del SIGN, se originó teniendo como foco de interés la temática del tratamiento y los procedimientos terapéuticos. Se diferencia de las anteriores por su particular énfasis en el análisis cuantitativo que aportan las $\mathrm{RS}$; y otorga además importancia a la reducción del error sistemático o sesgo (Figuras 10 y 11$)^{21}$.

Como fortaleza, es relevante destacar que considera la calidad metodológica de los estudios que componen las $\mathrm{RS}$, situación de sumo interés, dada la alta producción anual de RS.

Como debilidad podemos señalar que no considera en la elaboración de las recomendaciones la realidad científica y tecnológica del momento, pues éstas se crean con una rigidez que puede ser peligrosa para quienes usan con ortodoxia las recomendaciones para la implementación de políticas de salud. Por otro lado, se basa de forma puntual en los aspectos metodológicos y de diseño, pero no así en la dimensión de la perspectiva del padecer una enfermedad o las implicancias económicas de las medidas recomendadas; situación que puede limitar su utilización en la práctica clínica latinoamericana.

Antiguamente, el SIGN se basaba en los NE desarrolladas por la Agencia de E.U.A. para el Cuidado de la Salud y la Investigación (AHCPR). Dadas las limitaciones encontradas con este sistema, desarrollaron una nueva clasificación que utilizan desde el año $2000^{22}$.

Este sistema de clasificación pretende dar mayor peso a la calidad de la evidencia que respalda cada recomendación, y hacer hincapié en que el cuerpo de la evidencia debe ser considerado en su conjunto, y no depender de un sólo estudio para apoyar a cada recomendación. También pretende dar más peso a las recomendaciones respaldadas por estudios observacionales de gran calidad donde los EC no se pueden realizar por razones prácticas o éticas.

En 2009, el SIGN tomó la decisión de implementar el enfoque GRADE en su directriz metodológica, metodología que se encuentra actualmente en desarrollo ${ }^{21}$. 


\section{National Institute for Health and Clinical Excellence}

La iniciativa National Institute for Health and Clinical Excellence (NICE) nace del National Health Service del Reino Unido (NHS) y actualmente abarca la valoración de la evidencia en diferentes escenarios clínicos: tratamiento, diagnóstico, pronóstico y estudios de costo efectividad. Además incluye el tópico de la experiencia del paciente para informar preguntas de revisión (estudios cualitativos y encuestas en estudios transversales) y guías clínicas ${ }^{23}$.

Desde el año 2009 utiliza la pauta GRADE para evaluar la calidad de la evidencia en terapia y procedimientos terapéuticos. No obstante ello, NICE difiere de GRADE en dos puntos: integra una revisión de la calidad de los estudios de coste-efectividad; y no utiliza etiquetas como "resumen global" para la calidad de la evidencia o la fuerza de una recomendación, sino que utiliza la redacción de recomendaciones a fin de reflejar la fuerza de la recomendación.

Para valorar la calidad de la evidencia en estudios de diagnóstico, utiliza la herramienta QUADAS-2; y la calidad de la evidencia en estudios de pronóstico se realiza mediante la aplicación de una lista de chequeo ad-hoc. Por otra parte, para evaluar la calidad de las guías clínicas utiliza el instrumento AGREE II.

La jerarquización de la evidencia y los GR para estudios de pruebas diagnósticas se pueden apreciar en las Figuras 12 y 13.

Finalmente cabe señalar, que NICE presenta la información a través de un resumen de la calidad de la evidencia encontrada, el que es elaborado tras la aplicación de la evaluación sistemática de la información, utilizando para ello las herramientas ya mencionadas y la ulterior redacción de recomendaciones ${ }^{24}$.

\section{National Health and Medical Research Council}

El National Health and Medical Research Council (NHMRC) se ha utilizado en Australia desde 1999. Es una tabla de jerarquía de la evidencia, creada con el objetivo de valorar la evidencia en las guías de práctica clínica y evaluación de tecnologías sanitarias (Figura 14).

Los manuales proponen valorar la evidencia en tres dimensiones: la fuerza, el tamaño del efecto y la relevancia clínica. En este esquema, la solidez de la evidencia determina el nivel, la calidad de la evidencia y su precisión estadística. Con el uso de esta forma de jerarquización, se ha observado que a menudo la evidencia obtenida no es susceptible de ser sometida a meta-análisis y por tanto su valoración se relaciona sólo a los estudios individuales.

La tabla actual (basada en la jerarquización del CE$\mathrm{BM})$, es más amplia que la inicial y está estructurada de forma distinta. Contiene cinco columnas para cada una de las áreas de investigación (intervención, precisión

\begin{tabular}{|c|l|}
\hline $\begin{array}{c}\text { Nivel de } \\
\text { evidencia }\end{array}$ & Interpretación \\
\hline IA & RS con homogeneidad* de estudios de nivel $1^{\dagger}$ \\
\hline IB & Estudios de nivel $1^{\dagger}$ \\
\hline II & Estudios de nivel $2^{\ddagger}$ RS de estudios de nivel 2 \\
\hline III & Estudios de nivel $3^{\S}$ RS de estudios de nivel 3 \\
\hline IV & $\begin{array}{l}\text { Consenso, informes de comités de expertos u opiniones y/o experiencia clínica sin } \\
\text { valoración crítica explícita; o en base a la psicología, difusión de la investigación o } \\
\text { "principios básicos" }\end{array}$ \\
\hline $\begin{array}{l}\text { *Homogeneidad significa que no hay variaciones o estas son pequeñas en la dirección y grado de } \\
\text { los resultados entre los estudios individuales que incluye la RS. 'Estudios de nivel 1 son aquellos que } \\
\text { utilizan una comparación enmascarada de la prueba con un estándar de referencia validado, en una } \\
\text { muestra de pacientes que refleja a la población a quien se aplicaría la prueba. 'Estudios nivel } 2 \text { son } \\
\text { aquellos que presentan una sola de esta características: población reducida (la muestra no refleja las } \\
\text { características de la población a la que se le va a aplicar la prueba; utilizan un estándar de referencia } \\
\text { pobre (definido como aquel donde la 'prueba' es incluida en la 'referencia', o aquel en que las 'prue- } \\
\text { bas' afectan a la 'referencia'; la comparación entre la prueba y la referencia no está enmascarada; o } \\
\text { estudios de casos y controles. 'Estudios de nivel } 3 \text { son aquellos que presentan al menos dos o tres de } \\
\text { las características señaladas anteriormente. }\end{array}$ \\
\hline
\end{tabular}

Figura 12. Niveles de evidencia para estudios de pruebas diagnósticas. Propuesta de la NICE.

\begin{tabular}{|c|l|}
\hline $\begin{array}{c}\text { Grados de } \\
\text { Recomendación }\end{array}$ & Interpretación \\
\hline A & Estudios de pruebas diagnósticas con un nivel de evidencia la o Ib \\
\hline B & Estudios de pruebas diagnósticas con un nivel de evidencia II \\
\hline C & Estudios de pruebas diagnósticas con un nivel de evidencia III \\
\hline D & Estudios de pruebas diagnósticas con un nivel de evidencia IV \\
\hline
\end{tabular}

Figura 13. Grados de recomendación para estudios de pruebas diagnósticas. Propuesta de la NICE.

diagnóstica, pronóstico, etiología y tamizaje); y una columna ubicada al extremo izquierdo de las anteriores presenta los NE.

Al valorar la evidencia se sugiere indicar siempre el área de investigación, por ejemplo, NE II Intervención, NE IV prueba diagnóstica.

Es muy completa pero poco práctica para el uso cotidiano. Va acompañada de un glosario y un formulario tipo para registrar cada uno de los pasos de la valoración hasta la ulterior recomendación ${ }^{25}$.

\section{Practicing Chiropractors' Committee on Radiology Protocols}

El Practicing Chiropractors' Committee on Radiology Protocols (PCCRP) desarrolla guías clínicas, para lo cual utiliza una clasificación de NE adaptada de la clasificación utilizada por el United States Department of Health and Human Services ${ }^{26}$. Ésta, no se limita sólo a EC; sino que considera todos los tipos de estudios que pueden aportar evidencia para la práctica.

Además, el PCCRP utiliza la propuesta de GR presentada por Phillips y cols. ${ }^{27}$, con una leve modificación para ajustarse a los NE no clínicos (Figura 15).

De este modo, agrupa la evidencia en cinco niveles según el diseño de los estudios:

Nivel I. EC controlados con asignación aleatoria, incluyendo estudios cuasi-aleatorios como también con asignación alternada. 


\begin{tabular}{|c|c|c|c|c|c|}
\hline Nivel & Intervención & Precisión diagnóstica & Pronóstico & Etiología & $\overline{\text { Tamizaje }}$ \\
\hline $\mathrm{I}$ & RS de estudios nivel II & RS de estudios nivel II & RS de estudios nivel II & RS de estudios nivel II & RS de estudios nivel II \\
\hline II & EC controlado, con AA & $\begin{array}{l}\text { Estudios de precisión de } \\
\text { PD con una comparación } \\
\text { enmascarada e indepen- } \\
\text { diente con un estándar de } \\
\text { referencia válido, entre su- } \\
\text { jetos consecutivos con una } \\
\text { presentación clínica definida }\end{array}$ & $\begin{array}{l}\text { Estudios de cohortes pros- } \\
\text { pectivas }\end{array}$ & $\begin{array}{l}\text { Estudios de cohortes pros- } \\
\text { pectivas }\end{array}$ & EC controlado con AA \\
\hline III-1 & $\begin{array}{l}\text { EC pseudoaleatorizado controlado } \\
\text { (ej. asignación alternada o algún } \\
\text { otro método) }\end{array}$ & $\begin{array}{l}\text { Estudios de precisión de PD } \\
\text { con una comparación en- } \\
\text { mascarada e independiente } \\
\text { con un estándar de refe- } \\
\text { rencia válido, entre sujetos } \\
\text { no consecutivas con una } \\
\text { presentación clínica definida }\end{array}$ & Todo o ninguno & Todo o ninguno & $\begin{array}{l}\text { EC controlado pseudoaleatorizado } \\
\text { (por ejemplo, asignación alternada } \\
\text { o algún otro método) }\end{array}$ \\
\hline III-2 & $\begin{array}{l}\text { Estudios comparativo con contro- } \\
\text { les concurrentes: } \\
\text { - EC experimental sin AA } \\
\text { - Estudios de cohortes } \\
\text { - Estudios casos y controles } \\
\text { - Series temporales interrumpidas } \\
\text { con un grupo control } \\
\end{array}$ & $\begin{array}{l}\text { Comparación con un están- } \\
\text { dar de referencia que no } \\
\text { cumple con el criterio reque- } \\
\text { rido para el NE II y III-1 }\end{array}$ & $\begin{array}{l}\text { Análisis de los factores pro- } \\
\text { nósticos entre los sujetos } \\
\text { de un solo brazo de un EC } \\
\text { controlado con AA }\end{array}$ & $\begin{array}{l}\text { Estudios de cohortes retros- } \\
\text { pectivas }\end{array}$ & $\begin{array}{l}\text { Estudios comparativos con contro- } \\
\text { les concurrentes: } \\
\text { - Ensayo experimental sin AA } \\
\text { - Estudios de cohortes } \\
\text { - Estudios de casos y controles }\end{array}$ \\
\hline III-3 & $\begin{array}{l}\text { Estudios comparativos s/controles } \\
\text { concurrentes: } \\
\text { - Estudios con controles históricos } \\
\text { - EC Dos o más estudios de un } \\
\text { solo brazo } \\
\text { - EC Series temporales interrumpi- } \\
\text { das sin grupo control paralelo }\end{array}$ & $\begin{array}{l}\text { Estudios de casos y controles } \\
\text { de diagnóstico }\end{array}$ & $\begin{array}{l}\text { Estudios de cohortes retros- } \\
\text { pectivas }\end{array}$ & Estudios de casos y controles & $\begin{array}{l}\text { Estudios comparativos sin contro- } \\
\text { les concurrentes: } \\
\text { - Estudios con controles históricos } \\
\text { - EC Dos o más estudios de un } \\
\text { solo brazo }\end{array}$ \\
\hline IV & $\begin{array}{l}\text { Series de casos, ya sea con resulta- } \\
\text { dos post-test o pre-test/post-test }\end{array}$ & $\begin{array}{l}\text { Estudios de rendimiento } \\
\text { diagnóstico sin estándar de } \\
\text { referencia }\end{array}$ & $\begin{array}{l}\text { Series de casos, o estudios } \\
\text { de cohortes de sujetos en } \\
\text { diferentes etapas de la en- } \\
\text { fermedad }\end{array}$ & $\begin{array}{l}\text { Estudios de corte transversal } \\
\text { o series de casos }\end{array}$ & Series de casos \\
\hline
\end{tabular}

Figura 14. Niveles de evidencia según NHMRC.

\begin{tabular}{|c|c|c|}
\hline Tipo de estudios & Grados A - D & Grados a - d \\
\hline Nivel clínico I & $\mathrm{A}=$ Estudios nivel I consistentes ó RS ó meta análisis & \\
\hline Nivel clínico II & B= Estudios nivel II consistentes ó un sólo estudio nivel I & \\
\hline Nivel clínico III & B= Estudios nivel III consistentes & \\
\hline Nivel clínico IV & C= Estudios nivel IV consistentes ó extrapolaciones de nivel II o III & \\
\hline Opinión de experto $\mathrm{V}$ & $\mathrm{D}=$ Evidencia nivel $\mathrm{V}$ ó estudios inconsistentes de nivel I - IV & \\
\hline Estudio poblacional & & $\begin{array}{l}\mathrm{A}=\text { Estudios clase I consistentes } \\
\mathrm{B}=\text { Un solo estudio clase I o estudios consistentes clase II y III } \\
\mathrm{C}=\text { Estudios consistentes clase IV } \\
\mathrm{D}=\text { Evidencia no concluyente }\end{array}$ \\
\hline $\begin{array}{l}\text { Ciencias básicas, biomecánicos, } \\
\text { estudios de validez. } \\
\text { Encuestas profesionales }\end{array}$ & & $\begin{array}{l}A=\text { Estudios consistentes } \\
B=\text { Un solo estudio positivo } \\
D=\text { Estudios no concluyente }\end{array}$ \\
\hline Estudios de confiabilidad & & $\begin{array}{l}\mathrm{A}=\text { Estudios clase I consistentes } \\
\mathrm{B}=\text { Un solo estudio clase I o estudios consistentes clases II } \\
\mathrm{C}=\text { Un solo estudio clase II } \\
\mathrm{D}=\text { Evidencia no concluyente }\end{array}$ \\
\hline
\end{tabular}

Figura 15. Grados de recomendación PCCRP.

Nivel II. EC sin asignación aleatoria (estudio prospectivo, previamente planificado, con criterios de elegibilidad y medidas de resultado pre-determinados).

Nivel III. Estudios observacionales. Incluye estudios de cohortes prospectivos y retrospectivos, estudios de casos y controles; y la investigación de servicios de salud que incluya ajustes por posibles variables de confusión.

Nivel IV. Estudios observacionales sin grupo control (estudios de cohortes sin controles y series de casos).

$\mathrm{Y}$, nivel $\mathrm{V}$, que corresponde a la opinión de expertos. 


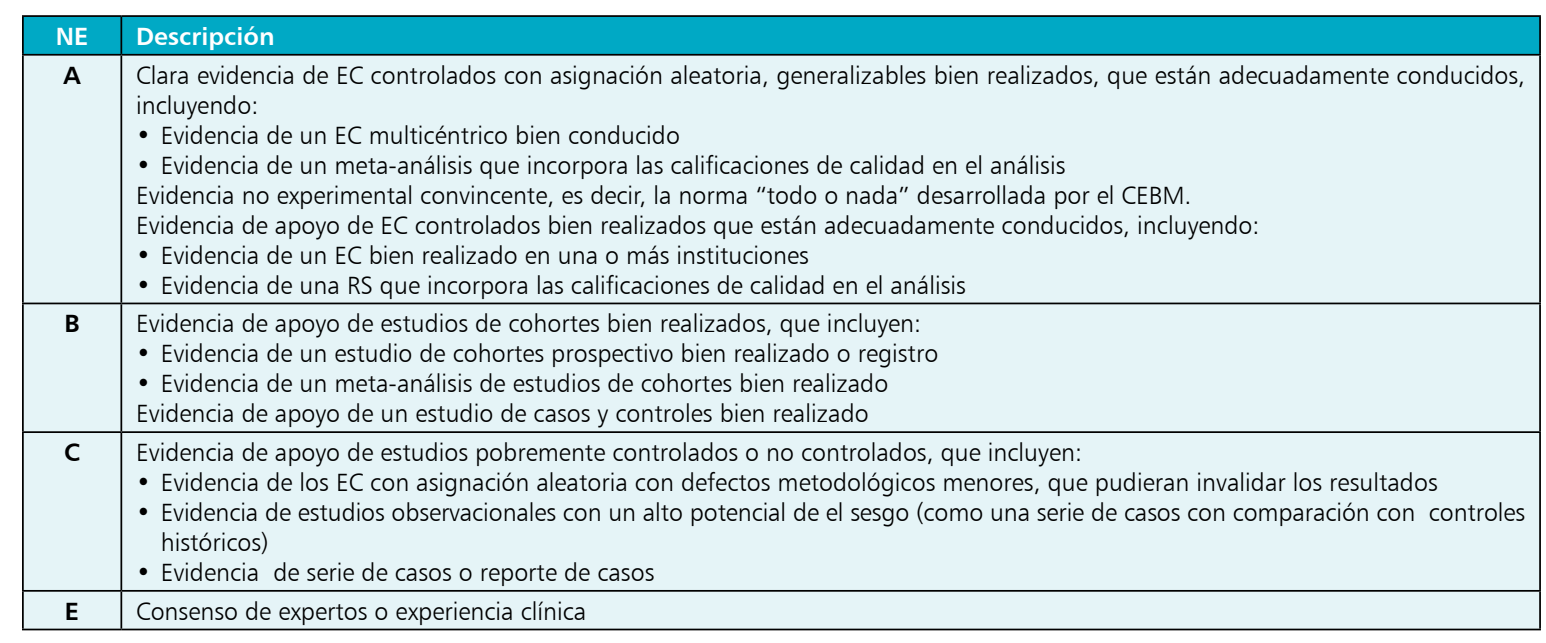

\section{American Diabetes Association}

La American Diabetes Association (ADA), ha participado activamente en el desarrollo y difusión de las normas de atención, directrices y documentos relacionados a la diabetes mellitus, aportando un sistema de clasificación para calificar la calidad de la evidencia científica que apoya sus recomendaciones disciplinarias (Figura 16) ${ }^{\mathbf{2 8}}$.

Estos niveles agrupados por letras, van de la $\mathrm{A}$ a la $\mathrm{E}$ $\mathrm{y}$ en base a esta valoración se realiza la recomendación.

Es una propuesta simple, en la que el NE ofrece directamente un GR, por ejemplo: un nivel A aporta un GR A.

Las recomendaciones se asocian a evidencia proveniente de estudios clínicos (niveles A-D). Por otra parte, un GR E se considera una categoría separada en las que no existe evidencia proveniente de EC; o, en situaciones en las que los que los EC pueden ser poco prácticos; $\mathrm{o}$, en aquellas circunstancias en las que hay evidencia contradictoria.

\section{The American College of Cardiology Foundation/ American Heart Association Task Force on Practice Guidelines}

El American College of Cardiology Foundation / American Heart Association (ACCF/AHA) Task Force es el encargado de elaborar, actualizar y revisar las directrices prácticas para las enfermedades cardiovasculares y sus respectivos procedimientos.

En el análisis de los datos y la elaboración de recomendaciones de las guías de práctica clínica, el comité de redacción utiliza metodologías basadas en la evidencia desarrolladas por el Task Force. La "clase de la recomendación" (COR) es una estimación del tamaño del efecto de un tratamiento; teniendo en cuenta los riesgos versus los beneficios, evidencia y grado de acuerdo que un determinado tratamiento o procedimiento es o no útil/ efectivo, o que en algunos casos puede causar daño ${ }^{29}$.
El esquema para COR y NE se resume en la Figura 17, que también ofrece frases sugeridas para escribir recomendaciones para cada COR.

Un aporte a esta metodología es la separación de las recomendaciones; por ejemplo: Clase III para delimitar si la recomendación es considerada "ningún beneficio", o se asocia con "daño" al paciente.

Por otro lado, dado el número cada vez mayor de estudios de comparación de efectividad, se sugieren verbos y frases de comparación para la redacción de recomendaciones para la eficacia comparativa de un tratamiento o estrategia frente a otro (pero sólo para COR I y IIa, NE A o B).

Lo interesante de este enfoque es que cualquier combinación de COR y NE es posible. Por ejemplo, una recomendación puede tener Clase I, incluso si se basa exclusivamente en la opinión de expertos y no hay estudios de investigación que se hayan llevado a cabo. Del mismo modo, una Clase IIa o IIb se le puede asignar un Nivel A, si hay varios EC controlados con asignación aleatoria que llegan a conclusiones divergentes.

Hacen énfasis en que la asignación de un NE B o C no debe interpretarse en términos que la recomendación es débil. Muchas de las preguntas clínicas importantes tratadas en las guías clínicas no se prestan a la experimentación o aún no han sido abordadas por las investigaciones de alta calidad. A pesar que los EC controlados con asignación aleatoria pueden no estar disponibles, la pregunta clínica puede ser tan relevante que sería negligente no incluirla en la guía.

\section{Discusión}

El paso fundamental para la aplicación clínica de la evidencia en torno a un problema clínico es darle un valor a la evidencia disponible que nos permita ejercer
Figura 16. Niveles de evidencia de la ADA. 


\begin{tabular}{|c|c|c|c|c|}
\hline & $\begin{array}{c}\text { Clase I } \\
\text { Beneficio }>>\text { > Riesgo } \\
\text { Procedimiento/tratamiento } \\
\text { DEBERIA ser realizado o } \\
\text { administrado }\end{array}$ & $\begin{array}{l}\text { Clase Ila } \\
\text { Beneficio > > Riesgo } \\
\text { Se requiere de estudios } \\
\text { adicionales con objetivos } \\
\text { focalizados. ES RAZONABLE } \\
\text { realizar un procedimiento o } \\
\text { administrar un tratamiento }\end{array}$ & $\begin{array}{c}\text { Clase Ilb } \\
\text { Beneficio } \pm \text { Riesgo } \\
\text { Se requiere de estudios } \\
\text { adicionales con objetivos } \\
\text { amplios; datos de registros } \\
\text { adicionales podrían ser de } \\
\text { ayuda. PODRIA CONSIDERARSE } \\
\text { el procedimiento o tratamiento }\end{array}$ & $\begin{array}{c}\text { Clase III } \\
\text { Riesgo } \pm \text { Beneficio } \\
\text { Procedimiento/tratamiento } \\
\text { NO debería ser realizado o } \\
\text { administrado PUESTO QUE } \\
\text { NO ES ÚTIL Y PUEDE SER } \\
\text { PERJUDICIAL }\end{array}$ \\
\hline $\begin{array}{l}\text { Nivel A } \\
\text { Múltiples poblaciones } \\
\text { evaluadas* } \\
\text { Datos derivados de múltiples } \\
\text { EC con AA ó RS }\end{array}$ & $\begin{array}{l}\text { - Recomendación: El tratamiento } \\
\text { es útil o efectivo } \\
\text { - Suficiente evidencia de múlti- } \\
\text { ples EC ó RS }\end{array}$ & $\begin{array}{l}\text { - Recomendación: a favor del } \\
\text { tratamiento o procedimiento } \\
\text { siendo útil o efectivo } \\
\text { - Alguna evidencia conflictiva de } \\
\text { múltiples EC ó RS }\end{array}$ & $\begin{array}{l}\text { - Recomendación de la utilidad/ } \\
\text { eficacia menos establecida } \\
\text { - Mayor evidencia contradictoria } \\
\text { de múltiples EC ó RS }\end{array}$ & $\begin{array}{l}\text { - Recomendación: El procedi- } \\
\text { miento o tratamiento no es útil } \\
\text { o efectivo y podría ser dañino } \\
\text { - Evidencia suficiente de múlti- } \\
\text { ples EC ó RS }\end{array}$ \\
\hline $\begin{array}{l}\text { Nivel B } \\
\text { Poblaciones limitadas } \\
\text { evaluadas* } \\
\text { Datos derivados de EC } \\
\text { simples o estudios sin AA }\end{array}$ & $\begin{array}{l}\text { - Recomendación: El procedi- } \\
\text { miento o tratamiento es útil o } \\
\text { efectivo } \\
\text { - Evidencia de un EC simple ó } \\
\text { estudios sin AA }\end{array}$ & $\begin{array}{l}\text { - Recomendación a favor del } \\
\text { tratamiento o procedimiento } \\
\text { siendo útil o efectivo } \\
\text { - Alguna evidencia contradictoria } \\
\text { proveniente de un solo EC ó de } \\
\text { estudios sin con AA }\end{array}$ & $\begin{array}{l}\text { - Recomendación de la utilidad/ } \\
\text { eficacia menos establecida } \\
\text { - Mayor evidencia contradictoria } \\
\text { de un solo EC ó estudios sin } \\
\text { AA }\end{array}$ & $\begin{array}{l}\text { - Recomendación: El procedi- } \\
\text { miento o tratamiento no es útil } \\
\text { o efectivo y podría ser dañino } \\
\text { - Evidencia de un solo EC ó de } \\
\text { estudios sin AA }\end{array}$ \\
\hline $\begin{array}{l}\text { Nivel C } \\
\text { Poblaciones muy limitadas } \\
\text { evaluadas* } \\
\text { Sólo consensos de opiniones } \\
\text { de expertos, estudios de } \\
\text { casos o de cuidados estándar }\end{array}$ & $\begin{array}{l}\text { - Recomendación: El procedi- } \\
\text { miento o tratamiento es útil o } \\
\text { efectivo } \\
\text { - Sólo opinión de expertos, es- } \\
\text { tudios de casos o de cuidados } \\
\text { estándar }\end{array}$ & $\begin{array}{l}\text { - Recomendación: a favor del } \\
\text { tratamiento o procedimiento } \\
\text { siendo útil o efectivo } \\
\text { - Sólo opinión de expertos con- } \\
\text { tradictoria, Estudios de casos o } \\
\text { de cuidados estándar }\end{array}$ & $\begin{array}{l}\text { - Recomendación de la utilidad/ } \\
\text { eficacia menos establecida } \\
\text { - Sólo opinión de expertos diver- } \\
\text { gente, Estudios de casos o de } \\
\text { cuidados estándar }\end{array}$ & $\begin{array}{l}\text { - Recomendación: El procedi- } \\
\text { miento o tratamiento no es útil } \\
\text { o efectivo y podría ser dañino } \\
\text { - Sólo opinión de expertos, Es- } \\
\text { tudios de casos o de cuidados } \\
\text { estándar }\end{array}$ \\
\hline
\end{tabular}

Figura 17. Niveles de evidencia del ACCF/AHA Task Force on Practice Guidelines.

el juicio clínico en base al grado de confiabilidad que los resultados de las investigaciones científicas nos entregan y que permita evaluar beneficios vs riesgos, a la luz de la evidencia actual.

Existen múltiples propuestas de clasificaciones para valorar la evidencia (se estima que superan el centenar); desde la inicial y más simple (Sackett), que sirvió de base para las actuales; hasta otras que han querido considerar todas las posibles situaciones que se pueden encontrar, haciéndolas inmanejables debido a su extensión. Entre otras tantas, se pueden mencionar las de la Agency for Healthcare Research and Quality ${ }^{30}$, de la Agència d'Avaluació de Tecnologia Mèdica (AATM) de la Generalitat de Catalunya ${ }^{31}$, de la Canadian Asthma Consensus Group $^{32}$; la de NANDA-I ${ }^{33}$, del Servicio Andaluz de Salud $^{34}$, SORT $^{35}$, etc.

Junto a ello, el lenguaje epidemiológico empleado, ha llevado consigo confusión e incomprensión por parte de los médicos clínicos, quienes ven en esta gran variedad de opciones más conflictos que ayuda al desarrollo de la práctica profesional. En este artículo se presentan las clasificaciones más relevantes existentes; utilizando como criterio de selección, el nivel de utilización de cada cual, el que se asocia directamente con el grado de aceptación de ellas por parte de la comunidad científica general o particular.

Sin embargo, lo interesante de todo esto, es que el desarrollo creciente de las guías clínicas y la realización de evaluaciones de tecnologías sanitarias, ha determinado la necesidad de utilización de cada una de las iniciativas antes señaladas, tanto en universidades como establecimientos sanitarios; razón por la que es relevante conocerlas, de modo tal de comprender mejor la metodología a través de la cual se efectúan estos procesos.

La sistematización de la búsqueda de la evidencia es el paso fundamental para la obtención de la mejor evidencia disponible. Sin embargo, al momento de acercarnos a ella siempre se ha de considerar la existencia de características poblacionales, culturales, económicas, tecnológicas, ambientales, etc.; es decir, darle relevancia al concepto de validez externa "sin importar" lo que "parece resultar" en otras latitudes, sin pruebas previas en nuestra realidad.

El paso siguiente corresponde a darle un valor a esta evidencia, para lo cual se debe elegir la clasificación que más se ajuste a nuestra necesidad y que permita discriminar entre un nivel y otro, para realizar las recomendaciones más adecuadas al entorno asistencial y poblacional. Es así como se dispone de clasificaciones amplias, con una propuesta distinta para diferentes escenarios (CEBM, NHMRC, etc.), como de clasificaciones más específicas, que apuntan a un escenario puntual (CTFPHC, SIGN, etc.) y otras como GRADE que ha sido incorporada para evaluar la calidad de la evidencia disponible, realizar recomendaciones y generar guías de práctica clínica basadas en la evidencia, por alrededor de 90 prestigiosas instituciones nacionales e internacionales ${ }^{10}$.

Finalmente, nos parece oportuno señalar, que este manuscrito aporta el resumen de la información referente a los 11 sistemas, propuestas y clasificaciones de jerarquización de la evidencia y GR de mayor uso en la actualidad 


\begin{tabular}{|c|c|c|c|c|c|c|c|c|c|}
\hline Clasificación & Tratamiento & Prevención & Etiología & Daño & Pronóstico & Diagnóstico & Prevalencia & Tamizaje & Económicos \\
\hline CTFPHC & & $x$ & & & & & & & \\
\hline SACKETT & $x$ & $x$ & $x$ & $x$ & $x$ & $x$ & & & $x$ \\
\hline USPSTF & & & & & & $x$ & & & \\
\hline CEBM & $x$ & $x$ & $x$ & $x$ & $x$ & $x$ & $x$ & & $x$ \\
\hline GRADE & $x$ & & & & & & & & \\
\hline SIGN & $x$ & & & & & & & & \\
\hline NICE & $x$ & & & & $x$ & $x$ & & & $x$ \\
\hline NHMRC & $x$ & & $x$ & & $x$ & $x$ & & $x$ & \\
\hline PCCRP & $x$ & & & & & & & & \\
\hline ADA & $x$ & & & & & & & & \\
\hline ACCF/AHA & $x$ & & & & & & & & \\
\hline
\end{tabular}

Figura 18. Clasificaciones de niveles de evidencia y grados de recomendación analizadas. Se puede observar que algunas de ellas son específicas para cierto tipo de escenarios.

(Figura 18); actualización de la información publicada en $2009^{6}$, documento en el que se plasmó la información relacionada a las seis propuestas de jerarquización más utilizadas en ese momento.

\section{Resumen}

Existen múltiples propuestas y clasificaciones que jerarquizan la evidencia, que pueden confundir a quienes se dedican a generar la evidencia tanto en evaluaciones de tecnología sanitaria, elaboración de guías clínicas, etc. El objetivo de este artículo es actualizar la información y describir las clasificaciones más utilizadas para valorar la evidencia en el ámbito de la salud, analizando sus principales diferencias y aplicaciones para que el usuario pueda elegir la que mejor se adapte a sus necesidades y tomar de este modo decisiones sanitarias basando su práctica en la mejor evidencia disponible. Se realizó una búsqueda sistemática de la literatura en las bases de datos PubMed y MEDLINE y en los buscadores Google, Yahoo e Ixquick. Se obtuvo una gran cantidad de información referente a niveles de evidencia y grados de recomendación, para finalmente resumir la información de 11 de las propuestas más utilizadas en la actualidad (CTFPHC, Sackett, USPSTF, CEBM, GRADE, SIGN, NICE, NHMRC, PCCRP, ADA y ACCF/AHA), entre las que destaca la del GRADE WORKING GROUP, incorporada por alrededor de 90 organizaciones nacionales e internacionales, tales como la World Health Organization, The Cochrane Library, American College of Physicians, American Thoracic Society, UpToDate, etc. y a nivel local por el Ministerio de Salud, para generar guías de práctica clínica.

\section{Referencias bibliográficas}

1.- Sackett D L, Rosenberg W M, Gray J A, Haynes R B, Richardson W S. Evidence based medicine: what it is and what it isn't. Br Med J 1996; 312: 71-2.

2.- Sackett D L, Richardson W S, Rosenberg W H R. Evidence-based medicine: how to practice and teach EBM. (Churchill Livingstone, 2000).

3.- Evidence-Based Medicine Working Group. Evidence-based medicine. A new approach to teaching the practice of medicine. JAMA 1992; 268: $2420-5$.

4.- Manterola C. Medicina basada en la evidencia o medicina basada en pruebas. Generalidades acerca de su aplicación en la práctica clínica cotidiana. Rev Med Clin Condes 2009; 20: 125-30.

5.- Manterola C. Medicina basada en la evidencia. Conceptos generales y razones para aplicación en cirugía. Rev Chil Cir 2002; 55: 550-4.
6.- Manterola C, Zavando D. (Grupo MINCIR). Cómo interpretar los "Niveles de Evidencia" en los diferentes escenarios clínicos. Rev Chil Cir 2009; 61: 582-95.

7.- Birtwhistle R, Pottie K, Shaw E, Dickinson J A, Brauer P, Fortin M, et al. Canadian Task Force on Preventive Health Care: we're back! Can Fam Physician 2012; 58: 13-5.

8.- Canadian Task Force on Preventive Health Care. New grades for recommendations from the Canadian Task Force on Preventive Health Care. Can Med Assoc J 2003; 169: 207-8.

9.- Canadian Task Force on Preventive Health Care. Putting prevention into practice. Grades of Recommendation, Assessment, Development, and Evaluation (GRADE) Working Group. Disponible en http://canadiantaskforce.ca/ methods/grade. (acceso el 10 de marzo de 2014).

10.- Guyatt G H, Oxman A D, Vist G E, Kunz R, Falck-Ytter Y, Alonso-Coello P, Schünemann
HJ; GRADE Working Group. GRADE: an emerging consensus on rating quality of evidence and strength of recommendations. Br Med J 2008; 336: 924-6.

11.- Sackett D L. Rules of evidence and clinical recommendations on the use of antithrombotic agents. Chest 1989; 95 (2 Suppl): 2S-4S.

12.- Harris R P, Helfand M, Woolf S H, Lohr K N, Mulrow C D, Teutsch S M, et al; Methods Work Group, Third US Preventive Services Task Force. Current methods of the US Preventive Services Task Force: a review of the process. Am J Prev Med 2001; 20 (3 Suppl): 21-35.

13.- U.S. Preventive Services Task Force. Grade Definitions. Disponible en http://www. uspreventiveservicestaskforce.org/uspstf/grades. htm. (acceso el 13 de febrero de 2014).

14.- Centre for Evidence-based Medicine (CEBM)-Levels of Evidence (March 2009). Disponible en http://www.cebm.net/index. aspx?o=1025. (acceso el 15 de febrero de 2014). 
15.- Centre for Evidence-based Medicine (CEBM)-Levels of Evidence (2011). Disponible en http://www.cebm.net/mod_product/design/ files/CEBM-Levels-of-Evidence-2.1.pdf. (acceso el 16 de febrero de 2014).

16.- Howick J, Chalmers I, Glasziou P, Greenhalgh T, Heneghan C, Liberati A, et al. Explanation of the 2011 Oxford Centre for Evidence-Based Medicine (OCEBM) Levels of Evidence (Background Document). (2011). Disponible en http://www. cebm.net/index.aspx?o=5653. (acceso el $16 \mathrm{de}$ febrero de 2014).

17.- Canfield S E, Dahm P. Rating the quality of evidence and the strength of recommendations using GRADE. World J Urol 2011; 29: 311-7.

18.- Guyatt G, Oxman A D, Akl E A, Kunz R, Vist G, Brozek J, et al. GRADE guidelines: 1 . Introduction-GRADE evidence profiles and summary of findings tables. J Clin Epidemiol 2011; 64: 383-94.

19.- Guyatt G, Gutterman D, Baumann M H, Addrizzo-Harris D, Hylek E M, Phillips B, et al. Grading strength of recommendations and quality of evidence in clinical guidelines: report from an American College of Chest Physicians Task Force. Chest 2006; 129: 174-81.

20.- SIGN. Methodological principles. Disponible en http://www.sign.ac.uk/methodology/index.html. (acceso el 18 de febrero de 2014).

21.- SIGN. SIGN 50 a guideline developer's handbook. (Scottish Intercollegiate Guidelines Network, 2011). Disponible en http://www.sign. ac.uk/guidelines/fulltext/50/index.html. (acceso el 18 de febrero de 2014).
22.- Harbour R, Miller J. A new system for grading recommendations in evidence based guidelines. Br Med J 2001; 323: 334-6.

23.- National Institute for Health and Care Excellence. NICE web page. Disponible en http://www.nice.org.uk. (acceso el 21 de febrero de 2014).

24.- NICE. The guidelines manual: consultation on the 2012 update. Disponible en http:// www.nice.org.uk/aboutnice/howwework/ developingniceclinicalguidelines/ GuidelinesManualConsultation2012.jsp. (acceso el 21 de febrero de 2014).

25.- Merlin T, Weston A, Tooher R. Extending an evidence hierarchy to include topics other than treatment: revising the Australian "levels of evidence". BMC Med Res Methodol 2009; 9: 34.

26.- PCCRP Document Section I. PCCRP web page. Disponible en http://www.pccrp.org. (acceso el 21 de febrero de 2014).

27.- Phillips B, Ball C, Sackett D, Badenoch D, Straus S, Haynes B D M: The Oxford Centre for Evidence-based Medicine Levels of Evidence. Levels of Evidence and Grades of Recommendation (2001). Disponible en http:// www.cebm.net/levels_of_evidence.asp. (acceso el 21 de febrero de 2014).

28.- American Diabetes Association. Introduction. ADA evidence-grading system for clinical practice recommendations. Diabetes Care 2008;31:S1-S2. Disponible en http://care. diabetesjournals.org/content/31/Supplement_1/ S1.full. (acceso el 22 de febrero de 2014).

29.- Greenland P, Alpert J S, Beller G A, Benjamin E J, Budoff M J, Fayad Z A, et al; American
College of Cardiology Foundation/American Heart Association Task Force on Practice Guidelines. 2010 ACCF/AHA guideline for assessment of cardiovascular risk in asymptomatic adults: executive summary: a report of the American College of Cardiology Foundation/American Heart Association Task Force on Practice Guidelines. Circulation 2010; 122: 2748-64.

30.- Agency for Healthcare Research and Quality (AHRQ). EPC Evidence Reports [Internet]. Rockville: AHRQ. Disponible en http://www. ahrq.gov/clinic/epcindex.htm\#methodology. (acceso el 19 de marzo de 2014).

31.- Jovell A J, Navarro-Rubio M D. Evaluación de la evidencia científica. Med Clin (Barc) 1995; 105: 740-3.

32.- Canadian Asthma Consensus Group. Disponible en http://www.lung.ca/cts-sct/pdf/Adult Asthma_Consensus.pdf. (acceso el 19 de marzo de 2014).

33.- NANDA-I. Disponible en http:// eldiagnosticoenfermero.blogspot.com. es/2010/12/niveles-de-evidencia-de-la.html. (acceso el 19 de marzo de 2014).

34.- Niveles de evidencia. Servicio Andaluz de Salud. Disponible en http://www. juntadeandalucia.es/servicioandaluzdesalud/ hrs3/index.php?id=manual_procedimientos niveles. (acceso el 19 de marzo de 2014).

35.- Ebell M H, Siwek J, Weiss B D, Woolf S H, Susman J, Ewigman B, et al. Strength of Recommendation Taxonomy (SORT): a patient-centered approach to grading evidence in the medical literature. Am Fam Physician 2004; 69: 548-56. 